\title{
Selective Periodic Component Carrier Assignment Technique in LTE and LTE-A Systems
}

\author{
Husnu S. Narman and Mohammed Atiquzzaman \\ School of Computer Science, University of Oklahoma, Norman, OK 73019 \\ Email: \{husnu, atiq\}@ou.edu
}

\begin{abstract}
Internet usage over mobile devices is on the rise. The bandwidth demand for mobile Internet access is also increasing with the number of mobile users. To answer users' demand, carrier aggregation is proposed in LTE-A system. In carrier aggregation, the best available one or more component carriers of each band are assigned to each user to provide efficient services. Several works have been reported in the literature on mandatory and periodic component carrier assignment methods. Although the previous works, especially periodic component carrier assignment methods, have significantly improved performance of LTE and LTE-A systems, many limitations still exist. One limitation of previous works is that data transfer is interrupted during periodic component carrier assignment operation which can decrease performance of the system. Therefore, in this paper, selective periodic component carrier assignment technique which allows continues data transfer during periodic carrier assignment operations is proposed. Results show that the proposed technique increases throughput rate up to $25 \%$ and decreases average delay time up to $35 \%$.
\end{abstract}

Index Terms-LTE, LTE-A, 4G, component carrier assignment, resources allocation, analysis.

\section{INTRODUCTION}

In LTE-A multi-band architecture, each band has several Component Carriers (CCs). User Equipment (UE) can simultaneously connect one or multiple carriers from different bands. Base stations (EnodeB) arrange the number of simultaneous connections of UEs from each band. Inefficient Component Carrier Assignment (CCA) significantly decreases system performance [1], [2].

There are several proposed component carrier assignment methods and their analysis [2]-[9] for LTE and LTE-A systems. In [3]-[6], full or partial feedback for Channel Quality Indicator (CQI) is used in order to find the best available carriers for each user. In [2], distribution of carriers to users are balanced. In [7], [8], service-based carrier assignment methods are proposed by giving priority for some services while assigning carriers to users. In [9], an uplink carrier assignment method has been proposed by considering a ratio function, traffic type and CQI to increase throughput while sending data from users to an eNodeB (eNB). While uplink carrier assignment methods try to optimize bandwidth and power usage, downlink carrier assignment methods try to optimize only bandwidth usage. In addition to the above carrier assignment methods, there exist traditional assignment methods, Least Load (LL) (LL can be called as Round Robin (RR)) and Random (R) methods [10]. LL method assigns users to least loaded carriers thus, LL method well balances users loads across carriers in short and long terms, and $\mathrm{R}$ method randomly selects carriers for users hence, $\mathrm{R}$ method only well balances users loads across carriers in long term. However, both of them ignore Quality of Service (QoS) requirements of each UE and CQI of channels. All of works [2]-[10] can be grouped under Mandatory Component Carrier Assignment (mCCA) methods which only update carriers based on mandatory changes (include path lost, CQI changes, etc).

In [11], a Periodic Component Carrier Assignment (pCCA) method is proposed and carriers are periodically assigned to each UE in specified time interval. Min-delay, higher CQI, etc. algorithms can be used in periodic carrier assignment methods in order to optimize delay or throughput of systems. For example, periodic carrier assignment method in [11] is a kind of min-delay-based method, which tries to minimize delay which is experienced by users.

In periodic carrier assignment, $\mathrm{CCs}$ of all users are updated periodically in addition to mandatory CCs assignment. As presented in [11], periodic carrier assignment significantly improves the performance of LTE and LTE-A systems. However, one known limitation of such system is interruption of data transfer during periodic carrier assignment process. This is due to reassignment all carriers of users at the same time in periodic carrier assignment [11]. Such technique can be called as Joint Periodic Component Carrier Assignment Technique ( $\mathrm{j}$-pCCA). The performance of periodic carrier assignment can be improved more because joint technique causes frequent packet transfer interruptions and that results delay and packet drops during periodic carrier assignment operations. Therefore, the aim of this work is to improve the performance of periodic component carrier assignment methods by eliminating frequent packet transfer interruptions.

The objective of this paper is to consider packet drops and delay which are experienced by users during periodic carrier assignment process and propose selective periodic carrier assignment technique (s-pCCA) to increase the performance of periodic carrier assignment methods for LTE and LTE-A systems. The key contributions of this work are as follows: (i) proposing selective periodic carrier assignment technique, (ii) the system models for joint and selective techniques are explained by using queuing system, (iii) comparing joint and selective techniques by applying both techniques to two well-known carrier assignment methods, modified LL and $\mathrm{R}$ (modified based on CQI and periodic assignment) with extensive simulation. $\mathrm{R}$ and LL methods are selected because 
of simplicity and common usage of them in the literature. Results show that the proposed technique increases throughput rate up to $25 \%$ and decreases average delay time up to $35 \%$ comparing to joint technique in the system. Our proposed technique and related analysis will help service providers build efficient periodic component carrier assignment methods in order to increase throughput and decrease average delay time.

The rest of the paper is organized as follows: In Section II, the system model of carrier assignment procedure for joint and selective techniques are explained and followed by queuing analysis of both techniques in Section III. Simulation environments with parameters are described in Section IV. In Section V, simulation results are presented and analyzed. Finally, Section VI has the concluding remarks.

\section{System Model with Joint and Selective TECHNIQUES}

Fig. 1 demonstrates a simple model of carrier assignment methods and packet schedulers. There are $n$ number of users and each user can only connect up to $m$ number of CCs. Today, LTE-A system can only support up to five simultaneous CCs connection for each user providing IMT-A level service [12]. One to two of CCs are primary component carriers for uplink and downlink, and can only be updated during handover [12], and the rest of carriers is Secondary Component Carriers (SCCs) which are updated for each user based on CQI of channels. However, as stated in [11], periodic carrier assignment is a new method try to reassign all CCs periodically in addition to mandatory carrier assignments. Therefore, both PCC and SCC are updated during the periodic carrier assignment operations for all users [11]. After carrier assignment process finishes,

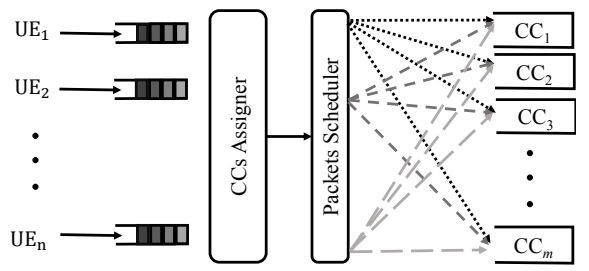

Fig. 1. General System Model with $n$ users and $m$ available CCs.

Packed Scheduler transfers packets over selected carriers in time and frequency domains. Currently Proportional Fairness and max-min are common packet schedulers in LTE systems [2], [11].

\section{A. Joint Periodic Component Carrier Assignment (j-pCCA)}

Mandatory carrier assignment methods allocate users to carriers based on mobility of users (including path and connection lost, low CQI, etc.). Therefore, when $\mathrm{UE}_{\mathrm{i}}$ moves from one position to another position, uplink and downlink carriers are updated to maintain connection. On the other hand, periodic carrier assignment methods allocate users to carriers based on time and periodically updates carriers in specified time intervals [11] regardless of users' mobility. During joint periodic carrier assignment process, all carriers are simultaneously updated for all users; packet transfer of users is thus interrupted. After joint periodic carrier assignment process is completed, packet transfer is recommenced.

\section{B. Proposed Selective Periodic Component Carrier Assign- ment (s-pCCA)}

As explained in Section II-A, disadvantage of joint technique is simultaneous reassignment of all carriers to users resulting in interruption of packet transfer. In order to provide better service, we have proposed a novel method, selective periodic carrier assignment, to solve the disadvantage of joint technique. In selective technique, only selected carriers of users are periodically updated. However, it is possible to update all carriers during selective periodic carrier assignment process according to selection algorithm.

Selective technique is taking into account time and CQI during periodic carrier assignment process in addition to Component Carrier Assignment Method. For example, LL method with selective technique is processed as follows for each periodic time:

- The threshold of CQI is predetermined for selection Algorithm (5 is selected as predetermined threshold for spCCA). Here, the threshold can be dynamically arranged by using user profile information for each user as is done in our past work [13].

- Partially or fully CQI feedback is obtained to measure the quality of CCs for each user. Note that, although CQI is low, the channel can transfer only a limited number of packets.

- The CCs, which have lower CQI than the predetermined threshold, are reseted and selected to be updated for each user.

- selective technique first selects new CCs, which have the least number of active users and have higher CQI than the threshold. It is very important to note that the number of new CCs may not be equal to previous number of CCs for each user. To make equal, more $\mathrm{CCs}$, which have the least number of active users, can be assigned. For example; assume that $\mathrm{UE}_{\mathrm{i}}$ receives data by using $C_{1}, C_{2}$, and $C_{3}$ component carriers and the $\mathrm{CQI}$ of $C_{1}$ and $C_{2}$ are lower than the threshold. Therefore, selective technique chooses $C_{1}$ and $C_{2}$ to update. However, selective technique only finds CQI of $C_{4}$ is higher than the threshold from all available CCs for $\mathrm{UE}_{\mathrm{i}}$. Therefore, $\mathrm{LL}$ method with selective technique assigns $C_{4}$ and the $\mathrm{CC}$, which has the least number of active users and the highest CQI (according to $\mathrm{UE}_{\mathrm{i}}$ ), to $\mathrm{UE}_{\mathrm{i}}$.

- To increase the efficiency and QoS, packet transferring priority is given to the $\mathrm{CC}$, which is the closest to the eNB.

Similarly, R method with selective technique is processed as above except that randomly assigning $\mathrm{CC}$ s from the CCs which have higher CQI than the threshold.

\section{ANALYSIS}

In this section, analytic expressions will be derived for performance metrics of joint and selective techniques during 
periodic carrier assignment by using queuing system.

\section{A. Notations}

The notations used for the analysis in the rest of the paper are listed in Table I.

TABLE I

NOTATIONS

\begin{tabular}{l|l}
\hline$i$ & $\in\{1,2, \ldots, n\}$ \\
\hline$j$ & $\in\{1,2, \ldots, m\}$ \\
\hline $\mathrm{Q}_{\mathrm{i}}$ & Queue of $\mathrm{UE} \mathrm{E}_{\mathrm{i}}$ \\
\hline$N$ & Size of each queue \\
\hline$p_{k}$ & Probability of $k$ number of packets in the system \\
\hline$\mu_{j}$ & Service rate of $\mathrm{CC}_{\mathrm{j}}$ \\
\hline$\lambda_{i}$ & Packet arrival rate of $\mathrm{UE}_{\mathrm{i}}$ \\
\hline$\delta$ & Avg. delay during periodic carrier assg. \\
\hline$n$ & Avg. queue length during periodic carrier assg. \\
\hline$D$ & Drop probability during periodic carrier assg. \\
\hline
\end{tabular}

B. Queuing Models of j-pCCA and s-pCCA for Downlink

Fig. 2 illustrates downlink process for $n$ users in LTE-A. The queuing model scheduler is Joint Queue Scheduler. We have used Joint Queue Scheduler because the performance of Joint Queue Scheduler is better than Disjoint Queue Scheduler for LTE-A [14]. While Joint Queue Scheduler allows all users to have disjoint buffers as showed in Fig. 2, Disjoint Queue Scheduler allows all CCs to have disjoint buffers.

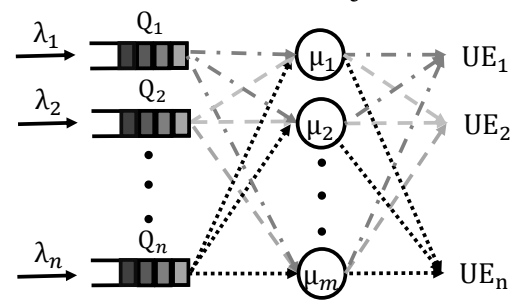

Fig. 2. Downlink Joint Queue Model with $n$ users and $m$ available CCs.

Downlink packet arrival rate for $\mathrm{UE}_{\mathrm{i}}$ is $\lambda_{i}$, each $\mathrm{CC}$ represented by a server and service rates of CCs are $\mu_{j}$ where $j \in\{1,2, \ldots, m\}$ and each buffer, $Q_{j}$, can hold at most $N$ packets. Packet schedulers enqueue an arrived packet which is requested by a user to one of assigned CCs. During joint periodic carrier assignment operation, packet transfer of $\mathrm{UE}_{\mathrm{i}}$ is terminated all the time. However, packet transfer of $U_{\mathrm{i}}$ is terminated if all carriers need to be updated or PCC needs to be updated during selective periodic carrier assignment operation (If PCC is updated then all carriers need to be updated). Therefore, there are two cases in the system for joint and selective techniques:

- Case 1: PCC is required to be updated, therefore SCCs are required to be updated (This case also includes when all carriers are required to be updated).

- Case 2: SCCs are required to be updated but PCC is not required to be updated.

The performance metrics of joint and selective techniques are same for Case 1. Hence, only Case 2 is explained to distinguish differences between joint and selective techniques. During periodic assignment operation (Case 2) in joint technique for $\mathrm{UE}_{\mathrm{i}}$, the packet transfer operation is as follows: (i)
Packet transfer is interrupted for the user. (ii) All servers of the user are updated. (iii) Packet transfer is recommenced for the user over new carriers. On the other hand, during periodic carrier assignment operations (Case 2) in selective technique, the process is as follows: (i) For all users, some carriers (CCs) are selected to be updated according to the selective algorithm (here, it is based on channel quality indicator) (ii) Packet transfer is only interrupted on carriers which are needed to be updated for each user. (iii) New carriers are assigned to users. (iv) Packet transfer is commenced on new carriers for the users.

\section{Assumptions}

To make the model analytically tractable, it is assumed that there is only one UE in the system as demonstrated in Fig. 3, all servers are capable of serving all type of packets, the queuing system is under heavy traffic flows, packet arrivals follow Poisson distribution, and service times for packets are exponentially distributed. Type of queue discipline used in

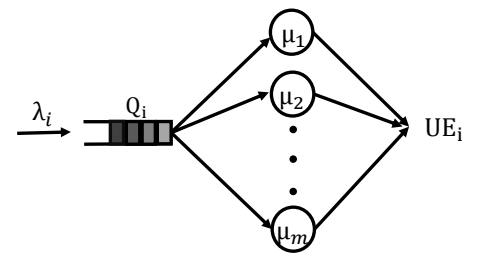

Fig. 3. Downlink Joint Queue Model with one user and $m$ available CCs.

the analysis is FIFO. Bandwidth and CQI of carriers can be different, so can service rates of all servers.

\section{Performance Metrics}

In this section, we will approximately derive drop probability, average queue length and average delay during periodic carrier assignment process for Case 2 in joint and selective techniques. In joint periodic carrier assignments, all carriers are updated for $\mathrm{UE}_{\mathrm{i}}$. Therefore, the service rate is zero and the system is not in steady state. Hence, we only mention the possibilities o performance of joint technique. On the other hand, we approximately derive performance metrics of selective technique.

1) State Probability: The service rate of the system is state dependent. When one packet is in the system, the service rate is $\mu_{t 1}=\mu_{1}$ and when two packets are in the system, the service rate is $\mu_{t 2}=\mu_{1}+\mu_{2}$. Service rate of the system increases until all carriers are utilized (c carriers for $\mathrm{UE}_{\mathrm{i}}$ ). Then the total server rate of the system is fixed at $\mu_{t c}$. It is important to note that there is at least one carrier which serves incoming traffic in selective technique which means $1 \leqslant c \leqslant m$. By using above approach, the state transaction diagram for selective techniques can be obtained as in Fig. 4. By using state transaction diagram, state probabilities can be obtained as follow:

$$
p_{k}= \begin{cases}p_{0} \frac{\lambda_{i}^{k}}{\prod_{v=1}^{k} \mu_{t v}} & k \leqslant c \\ p_{0} \frac{\mu_{t c}^{c} \rho^{k}}{\prod_{v=1}^{c} \mu_{t v}} & c<k \leqslant c+N\end{cases}
$$




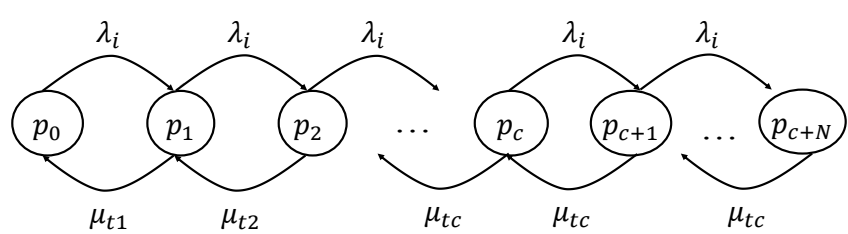

Fig. 4. State transaction diagram for the model.

where $\rho=\lambda_{i} / \mu_{t c}$. Using $\sum_{k=0}^{c+N} p_{k}=1$, we get

$$
p_{0}^{-1}= \begin{cases}1+\sum_{k=1}^{c} \frac{\lambda_{i}^{k}}{\prod_{v=1}^{k} \mu_{t v}}+\frac{\mu_{t c}^{c}}{\prod_{v=1}^{c} \mu_{t v}} \sum_{k=c+1}^{c+N} \rho^{k} & \rho \neq 1 \\ 1+\sum_{k=1}^{c} \frac{\lambda_{i}^{k}}{\prod_{v=1}^{k} \mu_{t v}}+N \frac{\mu_{t c}^{c}}{\prod_{v=1}^{c} \mu_{t v}} & \rho=1\end{cases}
$$

where $\rho=\lambda_{i} / \mu_{t c}$.

2) Drop Probability: Drop probability of the model is the final state probability which is $p_{c+N}$. Therefore, drop rate for selective technique can be obtained as follow:

$$
D=p_{0} \frac{\mu_{t c}^{c} \rho^{c+N}}{\prod_{v=1}^{c} \mu_{t v}}
$$

On the other hand, drop probability for joint technique can not be obtained because of unsteady state. It may be obtained by using $\lim _{\rho \rightarrow \infty} D$ or $\lim _{\mu \rightarrow 0} D$. Therefore, $D \approx 1$

3) Average Queue Length and Average Delay: Average queue length and average delay can be formulated by using state probability. Average queue length, $n$ for $M / M / 1 / N$ queue is as follows:

$$
n=\sum_{k=1}^{N} k p_{k}
$$

However, $M / M_{i} / c / N$ the system has $c$ servers and from the above state probabilities (Eqn. (1)), $n$ will be;

$$
n=\sum_{k=c+1}^{c+N}(k-c) p_{k}
$$

which gives the following expressions for $n$ :

$$
n= \begin{cases}p_{0} \frac{\mu_{t c}^{c}}{\prod_{v=1}^{c} \mu_{t v}} \rho^{c+1}\left(\frac{1-(N+1) \rho^{N}+N \rho^{N+1}}{(1-\rho)^{2}}\right) & \rho \neq 1 \\ p_{0} \frac{\mu_{t c}^{c}}{\prod_{v=1}^{c} \mu_{t v}}\left(\frac{N(N+1)}{2}\right) & \rho=1\end{cases}
$$

Using Little's law and Eqns. (3) and (6), average delay can be obtained as follows:

$$
\delta=\frac{n}{1-D}
$$

On the other hand, for joint technique, average queue length $(n)$ will be $n \approx N$. Therefore, average delay $(\delta)$ will be $\delta \approx \infty$. However, because periodic carrier assignment time duration is limited to a number (assume $\tau$ ), then $\delta=\tau$

\section{Simulation of The System}

In Section III, we approximately derive analytic performance metrics for selective technique and possible performance values for joint technique. We show that selective has improved performance of the system during periodic carrier assignment operations. However, overall system performance metrics can be different because service rate of carriers for each user are time and position dependent. Therefore, we have implemented simulation to observe the overall system performances of joint and selective techniques and show results in Section V.

\section{A. Assumptions for eNBs}

It is assumed that there is only one eNB which has three bands to provide service to users. The parameters of eNB is given in Table II.

TABLE II

eNB PARAMETERS

\begin{tabular}{l|l}
\hline Num. of eNB & 1 \\
\hline Used Bands & $800 \mathrm{MHz}, 1.8 \mathrm{GHz}, 2.6 \mathrm{GHz}$ \\
\hline Num. of CCs in Each Band & 4 \\
\hline Total Num. of CCs & 12 \\
\hline Queue Length of Each Queue & 50 packets \\
\hline Bandwidth of CCs & $10 \mathrm{MHz}$ \\
\hline Modulations & QPSK, 16QAM, and 64QAM \\
\hline CQI & $3,5,7$, and 11 \\
\hline Transmission Time Interval & $10 \mathrm{~ms}$ \\
\hline Time for CCA & $20 \mathrm{~ms}$ \\
\hline CQI Threshold & 5 \\
\hline
\end{tabular}

\section{B. Assumptions for UEs}

There are two types of equipment, LTE and LTE-A types in the system. Half number of equipment is LTE type and can only use one carrier and the other half number of equipment are LTE-A type and can use multiple carriers (up to five). In simulation, four CCs can be simultaneously used by LTEA type equipment because maximum five CCs can be used by LTE-A type equipment, and one of them must be used for upload primary component carriers (see Section II). Users are initially non-uniformly distributed in area which means that mostly users are located close to eNB. 50\% of users can move around of the eNB in specified time interval. Each user can only download one type of traffic. Packet arrivals follow Poisson distribution with 250 (packets per second) arrival rate for each user. And total arrival rates of traffic are enlarged when the number of users is increased. Selected Transmission Time Interval (TTI) for a packet is $10 \mathrm{~ms}$.

\section{Carrier Assignment Process and Packet Scheduling}

In simulation, modified LL and $\mathrm{R}$ methods are used for periodic carrier assignment by selecting carriers which have higher CQI than the threshold in addition to policies of LL and R methods. Therefore, during the simulation, LL method assigns carriers, which have the least number of users and have higher CQI than the threshold. If CQI of all of available carriers for a UE is not higher than the threshold, the least 


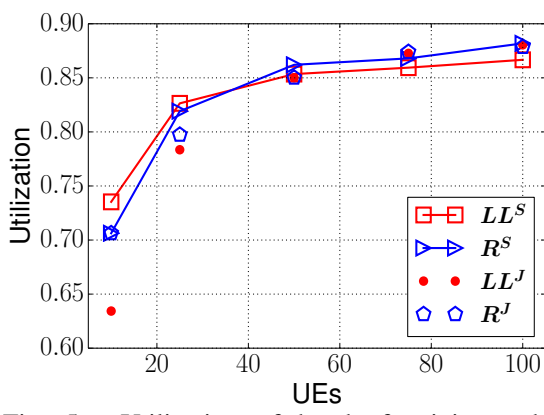

Fig. 5. Utilization of bands for joint and periodic techniques in R and LL methods.

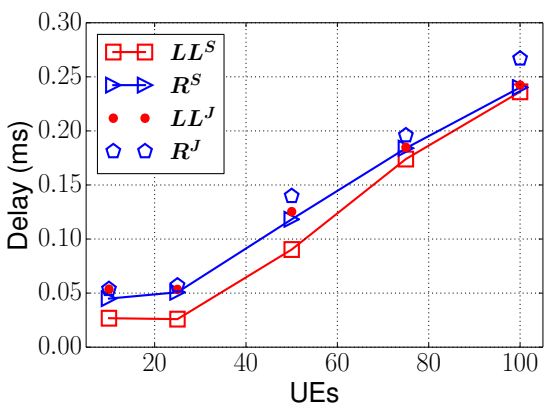

Fig. 6. Average delay of a packet for joint and periodic techniques in R and LL methods.

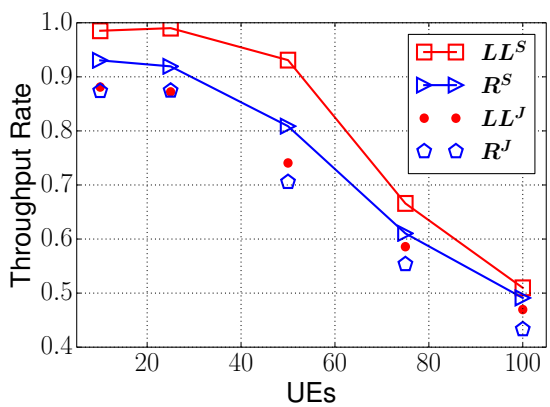

Fig. 7. Throughput rate for joint and periodic techniques in R and LL methods. loaded carriers are assigned to the UE. Similar ways are followed for $\mathrm{R}$ method in the simulation except that $\mathrm{R}$ method randomly assigns carriers to users.

We have used a min-delay packet scheduling method in order to compare joint and selective techniques. Packet arrival traffics are kept same for all test cases. Because of UEs and eNB positions, CQI Index for all carriers can be one of four options which are given in Table. II. Each packet is transferred by using one of assigned carriers. To increase the efficiency and QoS, packet transferring priority is given to the $\mathrm{CC}$, which is the closest to the eNB and minimizes packet delay if multiple carriers are available. If there is no available assigned carriers to serve arrived packets, packets are enqueued to corresponding user queues. If there is not any empty spaces in queues, arrived packets are dropped.

\section{Observation Methodology}

The results in Section V are average of 200 realizations for different size of users. The impact of light and heavy users loads on joint and selective techniques is investigated by using modified Random (R) and Least Load (LL) methods. R and LL methods are selected for test cases because of common usage in the literature and simplicity. Random periodic carrier assignment with joint technique $\left(R^{J}\right)$, Random periodic carrier assignment with selective technique $\left(R^{S}\right)$, Least Load periodic carrier assignment with joint technique $\left(L L^{J}\right)$, and Least Load periodic carrier assignment with selective technique $\left(L L^{S}\right)$ have been compared (Superscripts ${ }^{J}$ and $S$ in the figures represent joint and selective techniques, respectively).

\section{RESULTS}

In this section, overall system performance and experienced performance by each device type are presented for joint and periodic techniques.

\section{A. Overall Performance of the System}

In this subsection, the overall system performance for both LL and R methods with joint and selective techniques is presented by using band utilization, delay and throughput rate.

1) Utilization: Fig. 5 shows the utilization of bands for joint and selective techniques for LL and R methods. Utilization slowly increases when the number of users is higher than 25 for all cases. However, utilization is a suddenly increase for all cases when the number of user is changing from 10 to 25 .
That is because of increases in packet arrivals and the bands are not busy when the number of users is 10 . Though a large number of users (100 users), utilization of all cases does not reach peak rate $(=1)$ because all users are in the coverage area of Band-a but not in coverage areas of Band-b and Band-c. Thus, the utilization of bands does not reach peak rate although utilization of Band-a is high.

Moreover, there is no significant difference between band utilization of joint and selective techniques for $\mathrm{R}$ method except when the number of users is 25 or 50 . While the number of users is 25 or 50 , selective technique has higher band utilization than joint technique for $\mathrm{R}$ method. On the other hand, while the number of users are lower than 50, selective techniques has higher band utilization than joint technique for LL method and it is vice verse when the number of users is higher 50.

2) Delay: Fig. 6 demonstrates average delay per packet for joint and selective techniques. The average delay is increasing from 0.03 up to $0.27 \mathrm{~ms}$. While the number of users is growing, delay is regularly getting higher for all cases. This is due to high number of packet arrivals.

For both LL and R methods, selective technique has lower delay than joint technique. It is worth to mention that while the number of users is increased, the average delay gab between selective and joint is generally growing for $\mathrm{R}$ method and decreasing for LL method.

3) Throughput: Fig. 7 shows throughput rate for joint and selective techniques. The throughput rate is decreasing from 1 to 0.45 because increases in the number of users leads to gradually reduce throughput rate for all cases. For both LL and $\mathrm{R}$ methods, selective technique has higher throughput rate than joint technique. It is important to mention that the throughput gab between selective and joint techniques is almost same for $\mathrm{R}$ method except when the number of user is 50 . While the number of the users is 50, selective technique has much higher throughput rate than joint technique for $\mathrm{R}$ method. However, the throughput gab between selective and joint techniques is decreasing for LL method while the number of users is greater than 50 .

\section{B. Experienced Performance by Device Types}

We also investigate the experienced performance by device types (LTE and LTE-A device types). Subscripts ${ }_{L}$ and ${ }_{F}$ represent LTE and LTE-A type equipment, respectively in Figs. 8 


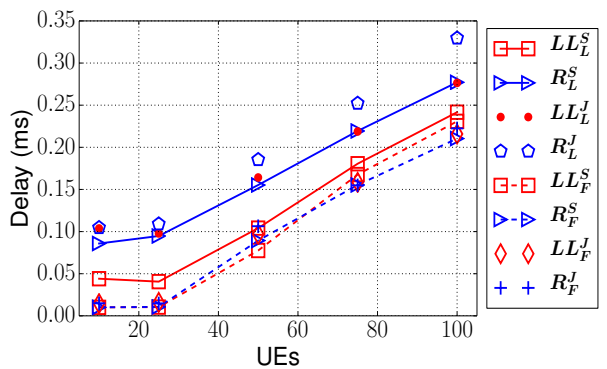

Fig. 8. Average delay of LTE and LTE-A type pakets for joint and periodic techniques in R and LL methods.

and 9. For example, $R_{L}^{S}$ represents Random periodic carrier assignment with selective technique for LTE type equipment and $R_{F}^{S}$ represents Random periodic carrier assignment with selective technique for LTE-A type equipment.

1) Delay: Fig. 8 shows average delay per packet which is experienced by LTE and LTE-A type equipment for joint and selective techniques. Average delay of LTE type equipment is higher than average delay of LTE-A type equipment for both $\mathrm{R}$ and LL methods because there is only one assigned CC to serve for LTE type equipment and multiple CCs for LTEA type equipment. Moreover, selective technique remarkably decreases average delay of LTE type equipment and slightly improves average delay time of LTE-A type equipment comparing to joint technique for both $\mathrm{R}$ and LL methods because of packet transfer interruption in joint technique.

2) Throughput Rate: Fig. 9 demonstrates throughput rate which is experienced by LTE and LTE-A types equipment for joint and selective techniques. Throughput rate of LTE type equipment is lower than throughput rate of LTE-A type equipment for both R and LL methods because of one $\mathrm{CC}$ assignment for LTE type devices. Furthermore, selective technique significantly increase throughput rate of LTE type equipment and slightly improves throughput rate of LTE-A type equipment for both $\mathrm{R}$ and LL methods.

\section{Summary of Results}

Based on the results, we make the following observations: (i) joint technique leads that LTE type equipment traffic suffers higher delay than LTE-A type equipment traffic, (ii) selective technique significantly enhances the performance for LTE and LTE-A type equipment, and (iii) selective technique remarkably decreases overall (up to 35\%) average delay and improve (up to $25 \%$ ) throughput rate comparing to joint technique in modified $\mathrm{R}$ and $\mathrm{LL}$ methods.

\section{CONCLUSION}

In this paper, selective periodic component carrier assignment technique is proposed by considering behavior of system during the component carrier assignment operations. The performances of current joint and proposed selective component carrier assignment techniques are compared by using analytic analysis based on queuing algorithm and an extensive simulation. Results show that the proposed technique efficiently uses system resources and improves throughput rate up to $25 \%$ and average delay time up to $35 \%$ in LTE and

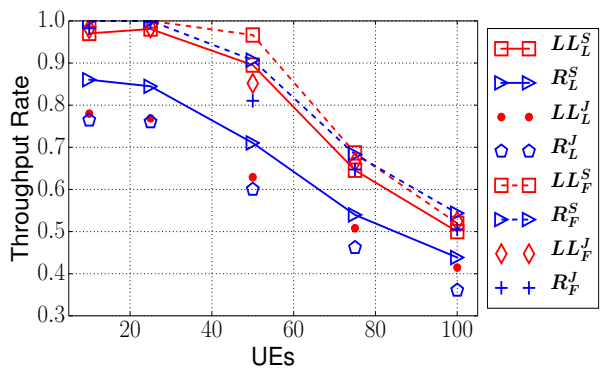

Fig. 9. Throughput rate of LTE and LTE-A type equipment for joint and periodic techniques in $\mathrm{R}$ and LL methods.

LTE-A systems. Our proposed technique and related analysis will help service providers build efficient periodic component carrier assignment methods in order to improve performances metrics such as throughput rate and delay.

\section{REFERENCES}

[1] I. F. Akyildiz, D. M. Gutierrez-Estevez, and E. C. Reyes, "The evolution to 4G cellular systems: LTE-Advanced," Physical Communication, vol. 3, pp. 217-244, Mar 2010.

[2] Y. Wang, K. Pedersen, T. Sorensen, and P. Mogensen, "Carrier load balancing and packet scheduling for multi-carrier systems," IEEE Transactions on Wireless Communications, vol. 9, no. 5, pp. 1780-1789, May 2010.

[3] L. xiang Lin, Y. an Liu, F. Liu, G. Xie, K. ming Liu, and X. yang Ge, "Resource scheduling in downlink LTE-Advanced system with carrier aggregation," The Journal of China Universities of Posts and Telecommunications, vol. 19, no. 1, pp. 44 - 49, Feb 2012.

[4] N. Kolehmainen, J. Puttonen, P. Kela, T. Ristaniemi, T. Henttonen, and M. Moisio, "Channel quality indication reporting schemes for UTRAN long term evolution downlink," in IEEE Vehicular Technology Conference, Singapore, May 11-14, 2008, pp. 2522-2526.

[5] S.-B. Lee, S. Choudhury, A. Khoshnevis, S. Xu, and S. Lu, "Downlink MIMO with frequency-domain packet scheduling for 3GPP LTE," in INFOCOM, Rio de Janeiro, Apr 19-25, 2009, pp. 1269-1277.

[6] S. Donthi and N. Mehta, "Performance analysis of subband-level channel quality indicator feedback scheme of LTE," in National Conference on Communications, Chennai, Jan 29-31, 2010.

[7] F. Liu, W. Xiang, Y. Zhang, K. Zheng, and H. Zhao, "A novel QoEbased carrier scheduling scheme in LTE-Advanced networks with multiservice," in Vehicular Technology Conference, Quebec City, Canada, Sep 3-6, 2012.

[8] W. Fu, Q. Kong, W. Tian, C. Wang, and L. Ma, "A QoS-aware scheduling algorithm based on service type for LTE downlink," in International Conference on Computer Science and Electronics Engineering, Hangzhou, China, Mar 22-23, 2013, pp. 2468-2474.

[9] R. Sivaraj, A. Pande, K. Zeng, K. Govindan, and P. Mohapatra, "Edgeprioritized channel- and traffic-aware uplink carrier aggregation in LTEAdvanced systems," in International Symposium on a World of Wireless, Mobile and Multimedia Networks, San Francisco, CA, June 25-28, 2012.

[10] T. Dean and P. Fleming, "Trunking efficiency in multi-carrier CDMA systems," in 56th Vehicular Technology Conference, Vancouver, Canada, Sep 24-28, 2002, pp. 156-160.

[11] X. Cheng, G. Gupta, and P. Mohapatra, "Joint carrier aggregation and packet scheduling in LTE-Advanced networks," in Communications Society Conference on Sensor, Mesh and Ad Hoc Communications and Networks, New Orleans, LA, June 24-27, 2013, pp. 469-477.

[12] J. Wannstrom. (2013, June) LTE-Advanced. Accessed: Mar. 18, 2015. [Online]. Available: http://www.3gpp.org/technologies/keywordsacronyms/97-lte-advanced

[13] H. Narman and M. Atiquzzaman, "Carrier components assignment method for LTE and LTE-A systems based on user profile and application," in IEEE GLOBECOM Workshop on Broadband Wireless Access, Austin, TX, Dec. 12, 2014.

[14] L. Chen, W. Chen, X. Zhang, and D. Yang, "Analysis and simulation for spectrum aggregation in lte-advanced system," in 70th Vehicular Technology Conference, Anchorage, AK, Sep 20-23, 2009. 